\title{
The societal influences and quality of life among healthcare team members under COVID-19 pandemic
}

\section{Wei-Tsung Kao}

Kaohsiung Municipal Kai-Syuan Psychiatric Hospital

\section{Su-Ting Hsu}

Kaohsiung Municipal Kai-Syuan Psychiatric Hospital

Frank Huang-Chih Chou (D f50911.tw@yahoo.com.tw)

Kaohsiung Municipal Kai-Syuan Psychiatric Hospital

\section{Li-Shiu Chou}

Kaohsiung Municipal Kai-Syuan Psychiatric Hospital

\section{Kuan-Ying Hsieh}

Kaohsiung Municipal Kai-Syuan Psychiatric Hospital

\section{Dian-Jeng Li}

Kaohsiung Municipal Kai-Syuan Psychiatric Hospital

Guei-Ging Lin

Kaohsiung Municipal Kai-Syuan Psychiatric Hospital

\section{Pay-Jen Wu}

Kaohsiung Municipal Kai-Syuan Psychiatric Hospital

\section{Wei-Jen Chen}

Kaohsiung Municipal Kai-Syuan Psychiatric Hospital Joh-Jong Huang

Kaohsiung Medical University Hospital

\section{Research Article}

Keywords: corona virus infection disease 2019 (COVID-19), quality of life, Short form-12 items (SF-12), Societal Influences Survey Questionnaire(SISQ), and Disaster-Related Psychological Screening Test(DRPST), Structural equation model (SEM)

Posted Date: April 6th, 2021

DOI: https://doi.org/10.21203/rs.3.rs-343362/v1 
License: (c) (i) This work is licensed under a Creative Commons Attribution 4.0 International License. Read Full License 


\section{The societal influences and quality of life among healthcare team members under COVID-19 pandemic}

Wei-Tsung $\mathrm{Kao}^{1,2}$;Su-Ting $\mathrm{Hsu}^{1,3}$; Frank Huang-Chih $\mathrm{Chou}^{{ }^{*}}$; Li-Shiu Chou ${ }^{1}$ Kuan-Ying

Hsieh $^{1,4}$; Dian-Jeng Li, MD ${ }^{1,5}$; Guei-Ging Lin ${ }^{1}$; Pay-Jen Wu ${ }^{1}$;Wei-Jen Chen ${ }^{1,3}$; Joh-Jong

Huang ${ }^{6,7^{*}}$

${ }^{1}$ Kaohsiung Municipal Kai-Syuan Psychiatric Hospital, Kaohsiung, Taiwan

${ }^{2}$ Department of Sports, Health and Leisure and Graduate Institute of Sports, Health and Leisure,

Cheng Shiu University, Kaohsiung, Taiwan

${ }^{3}$ Graduate Institute of Counseling Psychology and Rehabilitation Counseling, National

Kaohsiung Normal University, Kaohsiung, Taiwan.

${ }^{4}$ Graduate Institute of Medicine, College of Medicine, Kaohsiung Medical University, Kaohsiung,

Taiwan

${ }^{5}$ Department of Nursing, Meiho University, Pingtung, Taiwan

${ }^{6}$ Department of Family Medicine, Kaohsiung Medical University Hospital,

Kaohsiung City, Taiwan

${ }^{7}$ Department of Health, Kaohsiung City Government, Kaohsiung City, Taiwan

*contributed equally as corresponding authors

Address correspondence to:

Frank Huang-Chih Chou, M.D., Ph.D.

Department of Superintendent, Kaohsiung Municipal Kai-Syuan Psychiatric Hospital

No. 130, Kaisyuan 2nd Rd., Lingya District, Kaohsiung 802211, Taiwan

Tel: (886) 7-751-3171 ext. 2302

Fax: (886) 7-771-2494

Email: f50911.tw@yahoo.com.tw

OR 
Joh-Jong Huang, M.D., Ph.D.

Department of Health, Kaohsiung City Government

No.132-1, Kaisyuan 2nd Rd., Lingya District, Kaohsiung 802212, Taiwan

Tel: (886) 7-713-4000

Fax: (886) 7- 722-6940

E-mail: jjhua511227@gmail.com. 


\section{Abstract}

Background: The corona virus infection disease 2019 (COVID-19) pandemic is likely to put healthcare professionals across the world in an unprecedented situation.

Methods: 683 healthcare workers were recruited in this study. Short form-12 items (SF-12), Societal Influences Survey Questionnaire (SISQ), and Disaster-Related Psychological Screening Test (DRPST) were used to survey participants. Multiple linear regression and structural equation model (SEM) were used to explore the possible factors to the societal influences and quality of life.

Results: Female, older, more education years, married, regular intake and posttraumatic stress disorder (PTSD) frequency had positive effects to SISQ. To physical component summary (PCS) of SF-12, chronic illness, sleep score, PTSD frequency and social distance had negative effects and exercise habits had positive effect. To mental component summary (MCS) value of SF-12, age, participate in clubs, volunteer or charity activities and social information had positive effects and PTSD frequency, sleep score, social anxiety and depression had negative effects. Under SEM analysis, PTSD had positive effect to SISQ. Sleep score and MCS value had negative effects to SISQ. PTSD severity, older age, Sleep score, smoking and Nursing staff had negative effects to PCS value. Young age, PTSD frequency, sleep score and depression had negative effects to MCS value.

Conclusion: Healthcare team members with severer PTSD symptoms suffered more societal influences. Relative to PTSD severity, PTSD frequency was more important to the quality of life. Members of older age, who frequently participate in clubs, volunteers or charity activities had better mental life quality.

Keywords: corona virus infection disease 2019 (COVID-19), quality of life, Short form-12 items (SF-12), Societal Influences Survey Questionnaire(SISQ), and Disaster-Related Psychological Screening Test(DRPST), Structural equation model (SEM) 


\section{Background}

The COVID-19 pandemic is likely to put healthcare professionals across the world in an unprecedented situation, having to make impossible decisions and work under extreme pressures [1]. Among the healthcare workers also, they involved directly in handling these patients are at greater risk than others. The reasons for such adverse psychological outcomes in them range from excessive workload/work hours, inadequate personal protective equipment, over-enthusiastic media news, feeling inadequately supported [2, 3].

Another important reason for such psychological impact is the infection risk among medical staff. The sudden reversal of role from a healthcare worker to a patient might lead to frustration, helplessness, adjustment issues, stigma, fear of discrimination in the medical staff [4]. Despite the low mortality rate of $2 \%$, the COVID-19 virus has a high transmission rate and the mortality is higher than that caused by severe acute respiratory syndrome (SARS) and middle east respiratory syndrome (MERS) combined[5].

In past, there were several studies [6-8] about the association between mental health and biological disasters (like SARS, COVID-19) among healthcare members. A previous study reported that mental health problems were associated with social interaction and the ability to cope with COVID-19 among the general public in Taiwan [9].

In the face of such a biological disaster public health incidents like COVID-19, medical staff had massive stress on physical and mental health [10]. And past several studies [11-15] on the psychological impact of the SARS outbreak in Taiwan have focused on the psychiatric morbidity of medical professionals. In Taiwan's past experience in dealing with SARS, a study by a regional teaching hospital [16]showed that $17.3 \%$ of the medical staff involved in the care of SARS patients had obvious psychiatric symptoms, and the structural equation model (SEM) showed that the mother care and neuroticism had a direct impact on the ability of medical staff to deal with SARS, while mother control also had an indirect impact. A follow-up study[12] after 3 
years found that the medical staff with psychiatric symptoms showed that these symptoms were related to the stress of daily life and were less related to the SARS crisis. Compared with nurses, doctors have a higher ratio of physical symptoms, which indicates that different professionals suffered from different stress of mental health. In addition, it was found that early mothers parenting attitude and neuroticism had a greater impact on mental health while facing life-threatening stress.

Measuring the healthcare workers' risk perception of the COVID-19 and the relevant influential factors can provide the service providers, health policy makers as well as the health and hygiene instructors with great insights on facilitation of the behaviors aiming at self-effectiveness in improving the community health and selecting the best solutions for minimizing the risks arising from this disease. So the main aims of this study were (i) to investigate the possible factors to the societal influences and quality of life and (ii) to explore interrelationships and underlying mechanism between societal influences, mental life quality, physical life quality and related factors among the healthcare team members in a large psychiatric hospital under corona virus infection disease 2019 pandemic in Taiwan.

\section{Methods:}

\section{Subjects}

A cross-sectional investigation was used in the present study. 716 participants were collected from July 2020 to September 2020 at a psychiatric teaching hospital in southern Taiwan. Only 33 subjects did not complete questionnaires were found. A satisfactory response rate of $95.3 \%$ was thus obtained. Thus, this study consisted of 683 health care workers, including 44 physicians, 283 nurses, and 356 other hospital health care workers. The inclusion criteria were: 1) participants who worked at a large psychiatric hospital in southern Taiwan, 2) could understand the objective of the study and follow the instructions from research assistants, 3) aged 
between 20 to 65 years, and 3) informed consent was obtained from all subjects before filling in the questionnaire. Data with missing values or from those who could not complete the questionnaire were excluded. This study was approved by the Institutional Review Board of KSPH (KSPH-2020-03) and conducted according to the current revision of national legal requirements (Human Subjects Research Act, Taiwan).

Societal Influences, quality of life, Posttraumatic stress disorder (PTSD) symptoms, levels of depression, sleep disturbance and related demographic variables were collected through self-reported questionnaires.

T-test and Chi-square test were performed on the demographic variables and questionnaires between nursing staff and non-nursing staff. Multiple linear regression analyses were conducted to ascertain whether the independent factors were associated with dependent variables, including societal influences and quality of life (MCS and PCS). Structural equation model (SEM) was used to explore the possible factors to the societal influences and quality of life among healthcare team members in a psychiatric hospital by SEM. We also try to find out the associations of other latent variables and their relationship to PTSD scales (PTSD severity score and PTSD frequency score), level of depression and sleep disturbance by path analysis.

\section{Measures}

\section{Demographic data}

Data was recorded with the participants' age, educational years, marital status, gender, religion, types of work in the hospital, Smoking (yes or no), alcohol use ( $\geq 3$ times per week or not), Exercises habits ( $\geq 3$ days per week or not), Participate in clubs, volunteer or charity activities, Regular intake (three or four meals a day, $\geq 5$ days per week or not), chronic physical illness within 1 year, and History of hypertension and Diabetes. All of the demographic information was identified as categorical variables except age and educational years were continuous variables. 


\section{Societal Influences Survey Questionnaire (SISQ)}

The Societal Influences Survey Questionnaire (SISQ) was constructed to measure the psychological and social impact on individuals during the COVID-19 pandemic. With acceptable validity and reliability, the 15-item SISQ contains five categories of assessment: social distance, social anxiety, social desirability, social information, and social adaptation [17]. Ten of the questions were selected in the current study with four domains: social distance, social anxiety, social information, and social adaptation. Each question was scored on a 4-point Likert scale, with scores ranging from 1 (never) to 4 (often). Higher total scores of each domain (social distance, social anxiety, social information, and social adaptation) indicated higher compliance to maintain social distance, higher level of anxiety due to COVID-19, more desire to seek related information, and more awareness of progress of the pandemic overseas, respectively. Our past study [17] demonstrated that the SISQ has acceptable reliability, with Cronbach's alphas ranging between 0.57 and 0.76 . The SISQ accounted for $58.86 \%$ and satisfied the requirement of Kaiser-Mayer-Olkin values (0.78) and significant Bartlett's Test of sphericity. Moreover, the confirmatory factor analysis fit indices also indicated the adequacy of the model.

\section{Short form-12 items health survey $(S F-12)$}

The 12-item Short Form Survey version 2 (SF-12v2) is based on scoring coefficients derived from version 1 of the SF-36. It was developed to rapidly estimate general health status and has since been well validated [18]. The Short form-12 items health survey (SF-12v2)[19] is one of the most commonly used health-related quality of life (HRQoL) questionnaires, and it has become widely used in community-based health surveys and physical and mental illness outcome assessments due to its brevity and psychometric performance [20, 21]. The mental component summary (MCS) and physical component summary (PCS) exhibited high reliability (Cronbach's $\alpha>0.80$ ). A previous study [22] reported acceptable to good levels of reliability for PCS $($ ICC $=0.78)$ and MCS $($ ICC $=0.60)$. These items were graded on a 5-point Likert scale, 
with scores ranging from 1 (not at all) to 5 (extremely). A higher score represented a higher level of interference. And the questionnaire contained two components which were mental component summary (MCS) and physical component summary (PCS). So the score of SF-12v2 was divided into "PCS value of SF-12" and "MCS value of SF-12"

\section{Depression score}

Depression scales from the Disaster-Related Psychological Screening Test

The level of depression was measured using three questions from the Disaster-Related Psychological Screening Test (DRPST). The DRPST has been shown to be reliable and well-validated to rapidly screen for major depressive disorder or posttraumatic stress disorder (PTSD) after a disaster [23, 24]. Three items were used to estimate the status of depressed mood, fatigue or loss of energy, and worthlessness which had persisted for more than 2 weeks in the recent 1 month. Each item was rated on a 2-point Likert scale, with scores ranging from 0 (no) to 1 (yes). Higher total scores of the three items indicated higher levels of depression. Details of the questionnaire are listed in Supplementary Table S1.

\section{Sleep score}

Sleep disturbance scales from the Pittsburgh Sleep Quality Index

The Pittsburgh Sleep Quality Index (PSQI) was initially developed to measure the sleep quality in clinical populations, and it has been shown to have good validity and reliability [25]. Four items selected from the PSQI were used to estimate the level of sleep disturbance: difficulty to fall asleep, waking up in the middle of the night, subjective sleep quality, and enthusiasm in the recent 1 month (Supplementary Table S1). Each item was rated on a 4-point Likert scale, with scores ranging from 1 to 4 . Higher total scores of the four items indicated more severe sleep disturbance.

\section{Posttraumatic stress disorder (PTSD) symptoms}

Posttraumatic stress disorder (PTSD) scales from the Disaster-Related Psychological Screening 
Test

The levels of PTSD symptoms were measured using eight questions from the Disaster-Related Psychological Screening Test (DRPST). The DRPST has been shown to be reliable and well-validated to rapidly screen for major depressive disorder or posttraumatic stress disorder (PTSD) after a disaster [23, 24]. The PTSD scales divided into two components: PTSD severity score and PTSD frequency score. Eight items of PTSD severity and PTSD frequency were used to estimate the status of PTSD in the recent 1 month. Each item was rated on a 5-point Likert scale, with scores ranging from 1(PTSD severity: no distress; PTSD frequency: never) to 5(PTSD severity: extremely distress; PTSD frequency: everyday). Higher total scores of the eight items indicated higher levels of PTSD severity and PTSD frequency. Details of the questionnaire are listed in Supplementary Table S1.

\section{Statistical analysis}

T-test and Chi-square test were performed on the demographic variables and questionnaires between nursing staff and non-nursing staff. Marital status was transformed into a dichotomous variable as "married" (married and remarried) or "unmarried" (single, widowed, cohabiting and divorced). Histories of chronic illness were also transformed into dichotomous variables as "yes" or "no".

The data were analyzed using the IBM SPSS Statistics for Windows, Version 21.0 (IBM Corp. Released 2012. Armonk, NY: IBM Corp). Baseline characteristics and the scores of questionnaires were compared using an independent T-test or the chi-square test. Multiple linear regression analyses were conducted to ascertain whether the independent factors were associated with dependent variables, including SISQ total score_10, PCS value of SF-12, and MCS value of SF-12. The alpha level was set at 0.05 .

The normality of dependent variables was checked using the Kolmogorov-Smirnov test. Because non-normally distributed samples were identified with the significance of the test 
( $\mathrm{p}<0.001)$, bootstrapping multiple linear regression with 1000 bootstrap samples was used to verify the results from the stepwise multivariate linear regression analysis. In the bootstrapping method, $95 \%$ confidence intervals were used to determine statistical significance, as this could qualify the stability of the regression coefficients and reduce the length of the confidence interval [26]. When the $95 \%$ confidence interval of a regression coefficient did not contain zero, the variable was statistically significant. In addition, the number of bootstrap samples was set as 1000 to obtain sufficiently accurate $95 \%$ bootstrap percentile [27].

The AMOS 21.0 (SPSS, Chicago, IL, USA) was used to analyze the structural equation model (SEM). SEM uses the chi-square fit test to investigate the overall fit into the model; chi-squares resulting in $P$ value greater than 0.05 and an adjusted goodness-of-fit index greater than 0.9 indicated that the model adequately describes the observed data. Root Mean Square Error of Approximation (RMSEA) is based upon the non-centrality parameter. Good models have an RMSEA of 0.05 or less. Models whose RMSEA is 0.10 or less [28]. Furthermore, we explore the possible factors to the societal influences and quality of life among healthcare team members in a psychiatric hospital by SEM. We also try to find out the associations of other latent variables and their relationship to quality of life, PTSD scales, depression and sleep by path analysis.

\section{Results:}

716 participants were collected from July 2020 to September 2020 at a large psychiatric teaching hospital in southern Taiwan. Only 33 subjects did not complete questionnaires were found. A satisfactory response rate of $95.3 \%$ was thus obtained. Thus, this study consisted of 683 health care workers, including 44 physicians, 283 nurses, and 356 other hospital health care workers.

\section{Demographic data and questionnaires between Nursing staff and Non-Nursing staff.}

In table 1, we used T-test and Chi-square test to compare the difference of demographic data 
and questionnaires between two groups (Nursing staff group VS Non-Nursing staff group). We found that non-nursing staff group had more male than nursing staff group $\left(\chi^{2}=45.238, \mathrm{P}<0.001\right)$, non-nursing staff were older than nursing staff $(\mathrm{T}=97.569, \mathrm{P}<0.001)$, more non-nursing staff were married than nursing staff $\left(\chi^{2}=7.229, \mathrm{P}=0.007\right)$, more non-nursing staff had religion belief than nursing staff $\left(\chi^{2}=7.127, \mathrm{P}=0.008\right)$, more non-nursing staff had smoking than nursing staff $\left(\chi^{2}=13.593, \mathrm{P}<0.001\right)$, and more non-nursing staff had exercise habits $\left(\chi^{2}=38.018, \mathrm{P}<0.001\right)$, regular intake $\left(\chi^{2}=42.985, \mathrm{P}<0.001\right)$ and participate in clubs, volunteer or charity activities $\left(\chi^{2}=28.989\right.$, $\mathrm{P}<0.001)$ than nursing staff. More non-nursing staff had chronic physical illness than nursing staff $\left(\chi^{2}=5.384, \mathrm{P}=0.020\right)$ within 1 year, more non-nursing staff had hypertension than nursing staff $\left(\chi^{2}=4.752, \mathrm{P}=0.029\right)$, and more non-nursing staff had DM than nursing staff $\left(\chi^{2}=5.958, \mathrm{P}=0.015\right)$. There were no significant statistically difference over alcohol use $\left(\chi^{2}=0.032, \mathrm{P}=0.859\right)$ and education years $(\mathrm{T}=2.689, \mathrm{P}=0.015)$ between two groups. In the questionnaires, non-nursing staff group had less social anxiety $(\mathrm{T}=6.046, \mathrm{P}=0.014)$ and more social information $(\mathrm{T}=3.894$, $\mathrm{P}=0.049$ ) than nursing staff group. Non-nursing staff had less sleep disturbance than nursing staff under sleep score analysis $(\mathrm{T}=9.281, \mathrm{P}=0.002)$. Non-nursing staff had higher score of MCS value than nursing staff among SF-12v2 analysis. There were no significant statistically difference over SISQ total score $(\mathrm{T}=9.281, \mathrm{P}=0.002)$, social distance $(\mathrm{T}=9.281, \mathrm{P}=0.002)$, social adaption ( $\mathrm{T}=9.281, \mathrm{P}=0.002), \mathrm{PCS}$ value of $\mathrm{SF}-12 \mathrm{v} 2 \quad(\mathrm{~T}=9.281, \mathrm{P}=0.002)$, depression score $(\mathrm{T}=9.281$, $\mathrm{P}=0.002)$, PTSD frequency score $(\mathrm{T}=9.281, \mathrm{P}=0.002)$, and PTSD severity score $(\mathrm{T}=9.281$, $\mathrm{P}=0.002)$.

\section{Multiple linear regression for possible related factors of Total SISQ score}

After multiple linear regression analysis, we found that gender had positive effect on the SISQ total score.

After multiple linear regression (Table 2), we found that female $(\beta=0.143 ; \mathrm{P}<0.001$ ), older subjects $(\beta=0.113 ; \mathrm{P}=0.005)$, more education years $(\beta=0.103 ; \mathrm{P}=0.004)$, had marriage $(\beta=0.077$; 
$\mathrm{P}=0.049)$, regular intake $(\beta=0.109 ; \mathrm{P}=0.003)$, and $\mathrm{PTSD}$ frequency score $(\beta=0.313 ; \mathrm{P}<0.001)$ all had positive effects to SISQ score. After verification with 1000 bootstrapping multiple linear regressions, the significant related factors were the same as in multiple linear regression (Supplementary Table S4).

\section{Multiple linear regressions for possible related factors of PCS value of SF-12}

After multiple linear regression (Table 3), we found that chronic illness within 1 year $(\beta=-0.160 ; \mathrm{P}<0.001)$, Smoking $(\beta=-0.076 ; \mathrm{P}=0.031)$, sleep score $(\beta=-0.237 ; \mathrm{P}<0.001), \mathrm{MCS}$ value of SF-12 $(\beta=-0.151 ; \mathrm{P}<0.001)$, and PTSD frequency score $(\beta=-0.200 ; \mathrm{P}<0.001)$ and social distance $(\beta=-0.098 ; \mathrm{P}=0.007)$ all had negative effects to PCS value of SF-12. On the other hand, exercise habits $(\beta=0.147 ; \mathrm{P}<0.001)$ had positive effects to PCS value of SF-12. After verification with 1000 bootstrapping multiple linear regression, the significant related factors were the same as in multiple linear regression except smoking was excluded from bootstrapping methods (Supplementary Table S5).

\section{Multiple linear regression for possible related factors of MCS value of SF-12}

After multiple linear regression (Table 4), we found that age $(\beta=0.147 ; \mathrm{P}<0.001)$, Participate in clubs, volunteer or charity activities $(\beta=0.067 ; \mathrm{P}=0.026)$, and Social Information $(\beta=0.105 ; \mathrm{P}=0.002)$ all had positive effects to MCS value of SF-12. On the other hand, PTSD frequency score $(\beta=-0.265 ; \mathrm{P}<0.001)$, sleep score $(\beta=-0.211 ; \mathrm{P}<0.001)$, social anxiety $(\beta=-0.193$; $\mathrm{P}<0.001)$, depression score $(\beta=-0.232 ; \mathrm{P}<0.001)$, and PCS value of SF-12 $(\beta=-0.093 ; \mathrm{P}=0.003)$ all had negative effects to MCS value of SF-12. After verification with 1000 bootstrapping multiple linear regression, the significant related factors were the same as in multiple linear regression (Supplementary Table S6).

5. The Structural Equation Model (SEM) showing interrelationships between Societal Influences Survey Questionnaire (SISQ), mental component summary (MCS) value and physical component summary (PCS) value of Short form-12 items health survey (SF-12), PTSD, Age, Sleep score, 
depression score, Nursing Staff, gender, education years, and Smoking.

We used Structural Equation Model (SEM) analysis to explore interrelationships between Societal Influences Survey Questionnaire (SISQ), mental component summary (MCS) value and physical component summary (PCS) value of Short form-12 items health survey (SF-12), PTSD , Age, Sleep score, depression score, Nursing Staff, gender, education years, and Smoking. In Figure 1, we showed that PTSD (2 components: PTSD severity and PTSD frequency) had positive effect to SISQ (4 components: social information, social adaption, social distance, and social anxiety). Otherwise, sleep score and MCS value both had negative effect to SISQ.

Age had positive effect to social information and social distance. Education years had positive effect to social adaption. Nursing staff had positive effect to social anxiety.

PTSD severity had positive effect to sleep score and had negative effect to PCS value. PTSD frequency had positive effect to depression score and had negative effect to MCS value. Depression score and Nursing staff had positive effects to sleep score. Age had positive effect to MCS value and negative effect to PCS value. Sleep score and depression score both had negative effects to MCS value. Sleep score, Smoking and Nursing staff all had negative effects to PCS value.

Female had negative effect to smoking and positive effect to nursing staff. Nursing staff had negative effect to age. Age had negative effect to education years. Education years had negative effect to smoking.

\section{Discussion}

To the best of our knowledge, our study is the first study which focus on the related impact factors of societal influences, quality of life and investigate interrelationships between societal influences, mental health, physical health, posttraumatic stress disorder (PTSD), age, sleep score, depression score, nursing staff or not and smoking among healthcare team members in a large psychiatric hospital. In multiple linear regressions, PTSD frequency score had positive effects to 
SISQ score. In SEM analysis, PTSD also had positive effect to SISQ. However, Sleep score and MCS value both had negative effect to SISQ. So we can conclude that healthcare team member who had more severe PTSD symptoms suffered more societal Influences in a psychiatric hospital under COVID-19 pandemic. On the other hand, Health care team members who had poor sleep and mental health quality had less societal Influences in a psychiatric hospital under COVID-19 pandemic. Furthermore, multiple linear regression analysis for possible related factors of social distance, social anxiety, social information and social adaptation (four domain of SISQ) were used (Supplementary Table S7-S10). We found that PTSD frequency score had positive effects to four domain of SISQ. Sleep score and MCS value only had negative effect to social anxiety.

Depressive score had indirect negative effect to SISQ by sleep score and had indirect positive effect to SISQ by MCS value. So we cannot understand whether the healthcare team members with higher depression score would suffer from more societal Influences in a large psychiatric hospital under COVID-19 pandemic. Then we also used ANOVA test to compare the SISQ score between four groups (Depression score=0, 1, 2, 3, data showed at Supplementary Table S11), there was no significant statistical difference between four groups.

The major threat of the COVID-19 pandemic has seriously affected people's mental health $[9,29,30]$.And healthcare team members are also under such threats, their mental health has also been severely impacted[30, 31]. Our research focused on the differences in the mental health impacts among different types of healthcare team members who suffered threats in a psychiatric hospital under the COVID-19 pandemic. We found that there is no difference in their total SISQ scores regardless of whether they are nursing staff or not, but the score of nursing staff in social anxiety was higher, while the score of nursing staff in social information was lower, which shows that nursing staff is more anxious in the face of COVID-19 pandemic, but they receive less social information.COVID-19 had more effect on the mental health of non-nursing staff than nursing staff. On the other hand, we can find that the score of nursing staff in MCS 
value was lower, while the score of sleep score was higher, which showed that the mental health of nursing staff was better, but the sleep quality was worse. This may be related to the nature of the work that nursing staff required more shifts. Otherwise, a past study[12]about SARS in Taiwan showed that the major difference between the mental health of the nurses and the other healthcare workers was in the somatic realm (headache, palpitations, discomfort in the chest, and numbness of the limbs) in that the nurses had fewer complaints and symptoms. In our study, we can find that nursing staff were more female, younger, unmarried, less had religious belief, less smoking.

In the lifestyle analysis, we found that nursing staff had fewer exercise habits, less regular intake and less participation in clubs, volunteer or charity activities which may due to their working style. Most nursing staff requires shifts among their work that lead to poor lifestyle. Past study [32] showed that seventy nurses (63\%) worked nightshifts within the past year and poor sleep quality was the lifestyle factor which most strongly contributed to fatigue. Otherwise, we also found that nursing staff had less hypertension and diabetes which may due to their younger age.

In the multiple linear regression analysis, PTSD frequency score had positive effect to SISQ score and PTSD severity score had no effect to SISQ score. We can conclude that relative to PTSD severity score, PTSD frequency score was more important to the societal Influences among healthcare team members in a psychiatric hospital under COVID-19 pandemic. On the other hand, older female and married members suffered from more societal influences under COVID-19 pandemic. Health care team members with higher education level, regular intake suffered from more societal influences under COVID-19 pandemic. Past study [33] showed that culture factor also had influences on social distance under COVID-19 pandemic. But we can't consider the culture difference in a single-center study. Sleep score and MCS value only had negative effect to social anxiety, not affect other domains of SISQ. Another study [34] showed 
that medical staff had higher anxiety scores and depression scores than general population and the gender, age, marriage, working years, occupation, educational level, and economic income did not affect anxiety and depression. However, we did not investigate the related questionnaires among general population in our study.

In the multiple linear regressions, PTSD frequency score had a negative effect on MCS value and PCS value of Short form-12 items health survey and PTSD severity score had no effect on MCS value and PCS value. We can conclude that relative to PTSD severity score, PTSD frequency score was more important to the quality of life (physical and mental) among healthcare team members in a psychiatric hospital under COVID-19 pandemic. Otherwise, MCS value and PCS had effects on each other which mean there was a strong association between physical component and mental component of quality life. Members with sleep problem may worse their life quality among physical component and mental component, past study [35] also had similar finding. Members with chronic illness with 1 year, smoking and less exercise habits had poor life quality among physical component, like past studies [36-38]. Members with older age, participate in clubs, volunteer or charity activities frequently had better life quality on mental component. As past study [39, 40], depression can worse the life quality among mental component. Among the association between societal Influences and quality of life among healthcare team members in a psychiatric hospital under COVID-19 pandemic, members with higher social distance score had poor life quality among physical component. Members with higher social anxiety score had poor life quality among mental component. Members with higher social information score had better life quality among mental component.

\section{Limitation of the study}

First, societal influences and quality of life, other related factors like level of depression, sleep disturbance, PTSD were measured by self-reported questionnaires in our study, it would 
have been better if they had been verified through objective observations for related factors in our study. Second, the cross-sectional design of this study limited causal inference for further interpretation. Repeat measurement study may be considered to perform in the future. Finally, a single-center study may limit the generalizability and applicability to other populations.

\section{Conclusion}

Healthcare team members who had more severe PTSD symptoms suffered more societal Influences. Relative to PTSD severity score, PTSD frequency score was more important to the societal influences and quality of life among healthcare team members. On the other hand, older female and married members suffered from more societal Influences. Health care team members with higher education level, regular intake suffered from more societal Influences. Sleep problem may worse physical life quality and mental life quality, depression may worse mental life quality. Members with chronic illness with 1 year, smoking and fewer exercise habits had poor life quality among physical component. Members of older age, who frequently participate in clubs, volunteer or charity activities had better life quality among mental component. 


\begin{abstract}
Abbreviations
COVID-19:corona virus infection disease 2019; SF-12:Short form-12 items, SISQ :Societal Influences Survey Questionnaire; DRPST: Disaster-Related Psychological Screening Test; SEM Structural equation model; PTSD: posttraumatic stress disorder; PCS: physical component summary; MCS: mental component summary; SARS: severe acute respiratory syndrome; MERS: middle east respiratory syndrome; HRQoL: health-related quality of life; PSQI: Pittsburgh Sleep Quality Index

\section{Declarations}

Acknowledgements

This study was supported by Kaohsiung Municipal Kai-Syuan Psychiatric Hospital (KSPH) and approved by the Institutional Review Board of KSPH (KSPH-2020-03).

\title{
Author contributions
}

KWT: Conceptualization, Methodology, Formal analysis, Investigation, Writing - the main manuscript text, preparing all figures. HST: Formal analysis, Data curation, Conceptualization. CLS: Methodology, Data curation. HKY \& LDJ: Methodology, Investigation. LGG, WPJ \& CWJ: Investigation. CFHC: Conceptualization, Methodology, Formal analysis, Funding acquisition, Project administration, Writing - review \& editing. HJJ: Conceptualization, Formal analysis, Writing - review \& editing. All authors have read and approved the manuscript.

\section{Funding}

This study was not funded.

\section{Availability of data and materials}

The data that supports the findings of this study are available in the supplementary material of this article. And the further detail data of this study are available from the corresponding author upon reasonable request.

\section{Ethics approval and consent to participate}

This study was approved by the Institutional Review Board of KSPH (KSPH-2020-03) and 
conducted according to the current revision of national legal requirements (Human Subjects Research Act, Taiwan). Written informed consent was obtained from all subjects before filling in the questionnaires. Questionnaires were coded to prevent any identification to ensure study informants' anonymity and stored in the lockable cabinet except access to the study team to ensure confidentiality.

Consent for publication

Not applicable.

Competing interests

All authors declare that they have no conflicts of interest and are responsible for the content and writing of the paper. All authors have approved the final article. 


\section{References}

1. Greenberg, N., et al., Managing mental health challenges faced by healthcare workers during covid-19 pandemic. bmj, 2020. 368.

2. Cai, H., et al., Psychological Impact and Coping Strategies of Frontline Medical Staff in Hunan Between January and March 2020 During the Outbreak of Coronavirus Disease 2019 (COVID-19) in Hubei, China. Medical science monitor: international medical journal of experimental and clinical research, 2020. 26: p. e924171-1.

3. Lee, S.M., et al., Psychological impact of the 2015 MERS outbreak on hospital workers and quarantined hemodialysis patients. Comprehensive psychiatry, 2018. 87: p. 123-127.

4. Rana, W., S. Mukhtar, and S. Mukhtar, Mental health of medical workers in Pakistan during the pandemic COVID-19 outbreak. Asian journal of psychiatry, 2020. 51: p. 102080.

5. Mahase, E., Coronavirus: covid-19 has killed more people than SARS and MERS combined, despite lower case fatality rate. 2020, British Medical Journal Publishing Group.

6. Hsieh, K.-Y., et al., Mental health in biological disasters: From SARS to COVID-19. International journal of social psychiatry, 2020: p. 0020764020944200.

7. Hsu, S.-T., et al., Challenge and strategies of infection control in psychiatric hospitals during biological disasters -From SARS to COVID-19 in Taiwan. Asian Journal of Psychiatry, 2020: p. 102270.

8. Spoorthy, M.S., S.K. Pratapa, and S. Mahant, Mental health problems faced by healthcare workers due to the COVID-19 pandemic-A review. Asian journal of psychiatry, 2020. 51: p. 102119.

9. Li, D.-J., et al., COVID-19-related factors associated with sleep disturbance and suicidal thoughts among the Taiwanese public: A Facebook survey. International journal of environmental research and public health, 2020. 17(12): p. 4479.

10. Wu, P., et al., The psychological impact of the SARS epidemic on hospital employees in China: exposure, risk perception, and altruistic acceptance of risk. The Canadian Journal of Psychiatry, 2009. 54(5): p. 302-311.

11. Lu, Y.-C., et al., The mental health of hospital workers dealing with severe acute respiratory syndrome. Psychotherapy and psychosomatics, 2006. 75(6): p. 370-375.

12. Lung, F.-W., et al., Mental symptoms in different health professionals during the SARS attack: A follow-up study. Psychiatric quarterly, 2009. 80(2): p. 107.

13. Su, T.-P., et al., Prevalence of psychiatric morbidity and psychological adaptation of the nurses in a structured SARS caring unit during outbreak: A prospective and periodic assessment study in Taiwan. Journal of Psychiatric Research, 2007. 41(1-2): p. 119-130.

14. Chen, C.-S., et al., Psychological distress of nurses in Taiwan who worked during the outbreak of SARS. Psychiatric Services, 2005. 56(1): p. 76-79.

15. Chen, C., C. Kuo, and M. Lee, Effect of the severe acute respiratory syndrome epidemic on psychiatric morbidity of medical personnel. Taipei City Med J, 2004. 1(1): p. 75-80.

16. Lu, Y.-C., B.-C. Shu, and Y.-Y. Chang, The mental health of hospital workers dealing with severe acute respiratory syndrome. Psychotherapy and psychosomatics, 2006. 75(6): p. 370-375.

17. Li, D.-J., et al., Development and Implementation of Societal Influences Survey Questionnaire (SISQ) for Peoples during COVID-19 Pandemic: A Validity and Reliability Analysis. International journal of environmental research and public health, 2020. 17(17): p. 6246.

18. Fleishman, J.A., A.J. Selim, and L.E. Kazis, Deriving SF-12v2 physical and mental health summary scores: a comparison of different scoring algorithms. Quality of Life Research, 2010. 19(2): p. 231-241.

19. Huang, J.-J., et al., A preliminary report on psychiatric impairments and quality of life among Kaohsiung gas explosion victims 6 months after the event. Quality of life research, 2018. 27(3): p. 631-638.

20. Brazier, J.E. and J. Roberts, The estimation of a preference-based measure of health from the SF-12. Medical care, 2004: p. 851-859.

21. Burdine, J.N., et al., The SF-12 as a population health measure: an exploratory examination of potential for application. Health services research, 2000. 35(4): p. 885.

22. Cheak-Zamora, N.C., K.W. Wyrwich, and T.D. McBride, Reliability and validity of the SF-12v2 in the medical expenditure panel survey. Quality of Life Research, 2009. 18(6): p. 727-735.

23. Chou, F.-C., et al., The relationship between quality of life and psychiatric impairment for a 
Taiwanese community post-earthquake. Quality of Life Research, 2004. 13(6): p. 1089-1097.

24. Huang-Chih Chou, F., et al., Establishment of a disaster-related psychological screening test. Australian \& New Zealand Journal of Psychiatry, 2003. 37(1): p. 97-103.

25. Buysse, D., et al., Pittsburgh sleep quality index (PSQI). Psychiatry research, 1989. 28(2): p. 193-213.

26. Zhu, J. and P. Jing, The analysis of bootstrap method in linear regression effect. Journal of Mathematics Research, 2010. 2(4): p. 64.

27. Fox, J. and G. Monette, An R and S-Plus companion to applied regression. 2002: Sage.

28. Browne, M.W., et al., Alternative ways of assessing model fit. Sage focus editions, 1993. 154: p. 136-136.

29. Zandifar, A. and R. Badrfam, Iranian mental health during the COVID-19 epidemic. Asian journal of psychiatry, 2020. 51.

30. Torales, J., et al., The outbreak of COVID-19 coronavirus and its impact on global mental health. International Journal of Social Psychiatry, 2020: p. 0020764020915212.

31. Xiang, Y.-T., et al., Timely mental health care for the 2019 novel coronavirus outbreak is urgently needed. The Lancet Psychiatry, 2020. 7(3): p. 228-229.

32. Samaha, E., et al., Psychological, lifestyle and coping contributors to chronic fatigue in shift-worker nurses. Journal of advanced nursing, 2007. 59(3): p. 221-232.

33. Huynh, T.L.D., Does culture matter social distancing under the COVID-19 pandemic? Safety Science, 2020. 130: p. 104872.

34. Chen, Y., et al., Prevalence of self-reported depression and anxiety among pediatric medical staff members during the COVID-19 outbreak in Guiyang, China. Psychiatry research, 2020. 288: p. 113005.

35. Szentkirályi, A., C.Z. Madarász, and M. Novák, Sleep disorders: impact on daytime functioning and quality of life. Expert review of pharmacoeconomics \& outcomes research, 2009. 9(1): p. 49-64.

36. Berger, B.G. and A. McInman, Exercise and the quality of life. Handbook of research on sport psychology, 1993: p. 729-760.

37. Goldenberg, M., I. Danovitch, and W.W. IsHak, Quality of life and smoking. The American journal on addictions, 2014. 23(6): p. 540-562.

38. Taylor, R.M., F. Gibson, and L.S. Franck, A concept analysis of health-related quality of life in young people with chronic illness. Journal of clinical nursing, 2008. 17(14): p. 1823-1833.

39. Hohls, J.K., et al., Association between anxiety, depression and quality of life: study protocol for a systematic review of evidence from longitudinal studies. BMJ open, 2019. 9(3): p. e027218.

40. Shrestha, K., et al., Depression and its association with quality of life among elderly: An elderly home-cross sectional study. Neurology, Psychiatry and Brain Research, 2020. 38: p. 1-4. 


\section{Figures}

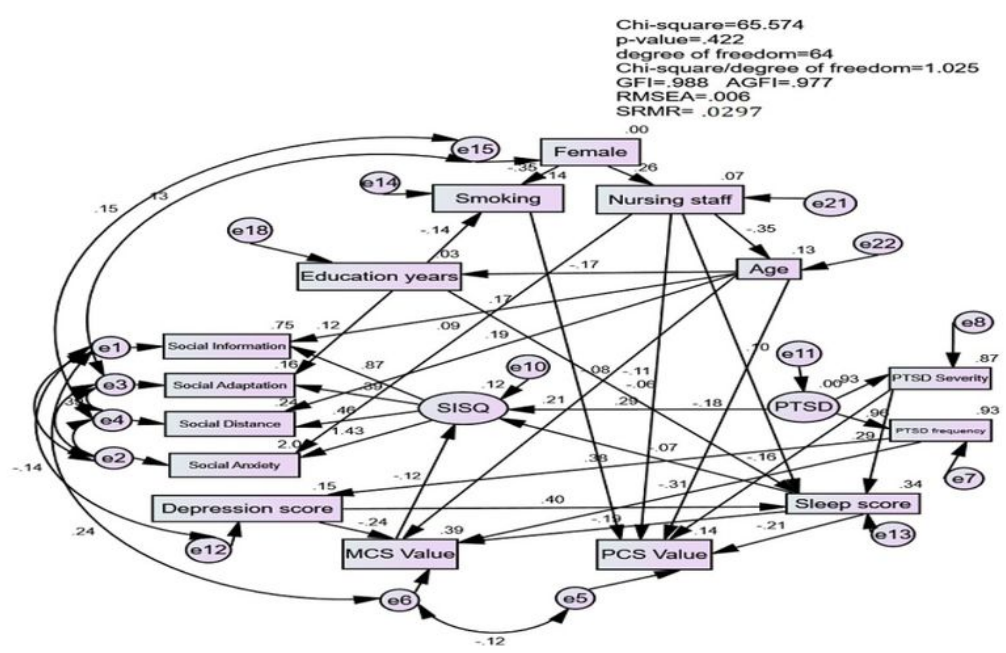

\begin{tabular}{|c|c|c|c|c|c|c|c|}
\hline & & & Estimate & $\begin{array}{l}\text { Standardixed } \\
\text { estimate }\end{array}$ & S.E. & C.R. & $P$ Value \\
\hline $\begin{array}{l}\text { PTSD frequency score } \\
\text {. }\end{array}$ & $\leftarrow$ & PTSD & 1.000 & 0.965 & & & \\
\hline PTSD Severity soore & $\leftarrow$ & PTSD & 0.824 & 0.930 & 0.034 & 24.162 & $<0.001$ \\
\hline Nursing Staff & $\leftarrow$ & Female & 0.283 & 0.249 & 0.039 & 7.193 & $<0.001$ \\
\hline Depression score & $\leftarrow$ & PTSD frequency score & 0.079 & 0.382 & 0.007 & 10.794 & $<0.001$ \\
\hline Age & $\leftarrow$ & Nursing Staff & -7.570 & -0.354 & 0.766 & -9.885 & $<0.001$ \\
\hline Education years & $\leftarrow$ & Age & -0.040 & -0.167 & 0.009 & 4.431 & $<0.001$ \\
\hline Sleep soone & $\leftarrow$ & Depression socre & 1.379 & 0.405 & 0.113 & 12218 & $<0.001$ \\
\hline Sleep soore & $\leftarrow$ & Nursing Staff & 0.527 & 0.098 & 0.168 & 3.131 & 0.002 \\
\hline Sleep soore & $\leftarrow$ & PTSD Severity score & 0.237 & 0.2808 & 0.027 & 8.687 & $<0.001$ \\
\hline Sleep soore & $\leftarrow$ & Education years & -0.067 & -0.063 & 0.033 & -2.034 & 0.042 \\
\hline MCS Value & $\leftarrow$ & Depression socre & -2.948 & -0.245 & 0.426 & -6.927 & $<0.001$ \\
\hline MCS Value & $\leftarrow$ & Sleep score & -0.665 & -0.188 & 0.126 & -5259 & $<0.001$ \\
\hline MCS Value & $\leftarrow$ & PTSD frequency score & -0.771 & -0.309 & 0.084 & -9.172 & $<0.001$ \\
\hline MCS Value & $\leftarrow$ & Age & 0.183 & 0.205 & 0.027 & 6.847 & $<0.001$ \\
\hline SISQ & $\leftarrow$ & PTSD & 0.112 & 0.295 & 0.016 & 6.837 & $<0.001$ \\
\hline Smoking & $\leftarrow$ & Female & -0.194 & -0.348 & 0.020 & -9.822 & $<0.001$ \\
\hline SISQ & $\leftarrow$ & MCS Value & -0.018 & -0.122 & 0.005 & -3.835 & $<0.001$ \\
\hline Smoking & $\leftarrow$ & Education years & -0.014 & -0.143 & 0.003 & -4.032 & $<0.001$ \\
\hline SISQ & $\leftarrow$ & Sleep score & -0.035 & -0.068 & 0.014 & -2.438 & 0.015 \\
\hline PCS Value & $\leftarrow$ & Sleep score & -0.461 & -0.211 & 0.086 & -5.345 & $<0.001$ \\
\hline Social Information & $\leftarrow$ & SISQ & 1.000 & 0.867 & & & \\
\hline Social Adaptation & $\leftarrow$ & SISQ & 0.529 & 0.387 & 0.117 & 4.525 & $<0.001$ \\
\hline Social Distance & $\leftarrow$ & SISQ & 1.005 & 0.458 & 0214 & 4.705 & $<0.001$ \\
\hline Social Anxiety & $\leftarrow$ & SISQ & 1.729 & 1.426 & 0211 & 8.197 & $<0.001$ \\
\hline PCS Value & $\leftarrow$ & Age & -0.099 & -0.180 & 0.021 & 4.743 & $<0.001$ \\
\hline PCS Value & $\leftarrow$ & Nursing Staff & -1.342 & -0.114 & 0.448 & -2.996 & 0.003 \\
\hline PCS Value & $\leftarrow$ & Smoking & -1.878 & -0.078 & 0854 & -2200 & 0.028 \\
\hline PCS Value & $\leftarrow$ & PTSD Severity score & -0288 & -0.160 & 0.071 & 4.069 & $<0.001$ \\
\hline Social Information & $\leftarrow$ & Age & 0.026 & 0.170 & 0.005 & 5.155 & $<0.001$ \\
\hline Social Distance & $\leftarrow$ & $\mathrm{Age}_{\mathrm{ge}}$ & 0.053 & 0.187 & 0.009 & 5848 & $<0.001$ \\
\hline Social Adaptation & $\leftarrow$ & Education years & 0.086 & 0.116 & 0.024 & 3.637 & $<0.001$ \\
\hline Social Anriety & $\leftarrow$ & Nursing Staff & 0.307 & 0.090 & 0.101 & 3.039 & 0.002 \\
\hline
\end{tabular}

Figure 1

The conceptual model showing interrelationships between Societal Influences Survey Questionnaire (SISQ) , mental component summary (MCS) value and physical component summary (PCS) value of Short form 12 items health survey (SF 12v2), PTSD , Age, Sleep score, depression score, Nursing Staff, gender, education years, and Smoking

\section{Supplementary Files}

This is a list of supplementary files associated with this preprint. Click to download.

- ThesocietalinfluencesandqualityoflifeamonghealthcareteammembersunderCOVID19pandemicTables1100216.pdf

- ThesocietalinfluencesandqualityoflifeamonghealthcareteammembersunderCOVID19pandemicSupplementarymaterial1100216.pdf 